\title{
Kinetics of Electrode Processes on Metals Coated with Hydrocarbon Films in Solutions of Electrolytes
}

\author{
V. I. Vigdorovitch ${ }^{1,2}$, L. E. Tsygankova ${ }^{2, *}$, M. Vigdorowitsch ${ }^{4}$, \\ N. V. Shel ${ }^{3}$ and L. G. Knyazeva ${ }^{1}$ \\ ${ }^{I}$ Russian Scientific Research Institute of Use of Machinery and Oil Products, \\ Tambov, 392000, Russia \\ ${ }^{2}$ Derzhavin State University, Tambov, 392000, Russia \\ ${ }^{3}$ Tambov State Technical University, Tambov, 392000, Russia \\ ${ }^{4}$ Angara GmbH, Düsseldorf, D-40627, Germany \\ *Corresponding author: vits21@ mail.ru
}

Received 22/11/2018; accepted 05/03/2021

https://doi.org/10.4152/pea.2021390503

\begin{abstract}
Kinetics regularities of hydrogen evolution reaction (HER) on Armco iron and copper anodic ionization, covered with a hydrocarbon film based on I-20A oil with a fixed amount of gun grease of the highest quality (GGHQ), were studied at room temperature. The measurements were carried out in aqueous and methanol solutions with the electrolyte composition of $\mathrm{x} \mathrm{M} \mathrm{HCl}+(1-\mathrm{x}) \mathrm{M} \mathrm{LiCl}$ and $0.1 \mathrm{M} \mathrm{HCl}+\mathrm{x} \mathrm{M} \mathrm{LiCl}+(3.9-$ x) $\mathrm{M} \mathrm{LiClO}_{4}$. The Tafel slope coefficients values, and the orders of reactions, with respect to hydrogen ions $(\mathrm{Fe}, \mathrm{Cu})$ and chloride-ions $(\mathrm{Cu})$ were estimated. The effect of oxyethylated amines (OEA), introduced into the solution or hydrocarbon surface coating, on the kinetics of electrode processes, for iron and copper, has been studied. It is shown that HER mechanism on Armco iron and copper anodic ionization does not change in the presence of a hydrocarbon coating on the metal surface. Large OEA molecules freely penetrate from the solution through the hydrocarbon coating to the metal surface, changing partial electrode reactions kinetics. The results are interpreted taking into account the hydrocarbon films surface porous structure.
\end{abstract}

Keywords: kinetics, electrode processes, iron, copper, oil coating, discontinuities.

\section{Introduction}

From the middle of XX century to the present, in the corrosive literature, it has been widely recommended to use hydrocarbon films, formed by products obtained from petroleum oils, as anticorrosive materials $[1,2]$. Such an approach makes it possible to substantially reduce the cost of compounds that protect surfaces, if they are sufficiently effective. While interpreting the protective effectiveness nature of oil-based compositions, the author [1] proceeded primarily from the fact that hydrocarbon viscous liquid-phase films, such as well wetting agents (Table 1), totally displace the phase and adsorption films of moisture from the metal surface. 
In this case, the absence of water, as a potential reagent in metals corrosion processes, substantially inhibits them.

However, this approach is not entirely correct. First, water and other corrosion stimulators $\left(\mathrm{O}_{2}, \mathrm{SO}_{2}\right)$ pass through such coatings with a thickness of $(5-6) 10^{4} \mathrm{~nm}$, without hindrance. Moreover, this process does not decay with time [3, 4].

Table 1. Values of contact angles $\left({ }^{\circ}\right)$ on steel and copper surfaces (authors' data).

\begin{tabular}{|c|c|c|}
\hline Nature of the liquid phase & Iron Armco & 1 M copper \\
\hline Distilled water & 77 & 82 \\
0,5 M NaCl solution & 77 & 81 \\
Aqueous extract of oil with 1 wt. \% GGHQ composition & 73 & 74 \\
Aqueous extract of oil with 10 wt. \% GGHQ* composition & 73 & 72 \\
I-20A Oil & 11 & 8 \\
n-heptane & $\sim 0$ & $\sim 0$ \\
n-decane & $\sim 0$ & 0 \\
\hline
\end{tabular}

* Technical substance: gun grease of the highest quality.

It has been experimentally shown that water, as a reagent of conjugated cathodic and anodic reactions, through hydrocarbon films, does not determine their rate. At the same time, any research, which it would be noted, on the fact that metals corrosion, under such conditions, is non-electrochemical, and that partial electrode reactions would not obey electrochemical kinetics laws, seems to be absent $[3,4]$. If there would be no aqueous electrolyte solution, in one form or another (own phase, micellar systems), under the hydrocarbon film, it would be difficult to imagine the formation of a double electrical layer at the solid/liquid interface. Then, the development of potential-controlled electrode reactions and electrochemical metals corrosion would be impossible. The liquid hydrocarbons dielectric constant, $\mathcal{E}$, is known to be close to 2 [3]. Consequently, in the hydrocarbon phase, there is no electrolytic dissociation of substances with an ionic, polar covalent, or any other similar to that, of bond nature. This, inevitably, leads to the electrochemical corrosion absence, and suppresses the formation of a double electric layer at the solid/liquid interface, although such substances are electrolytes in aqueous media, and other solvents with high $\varepsilon$ values. The specific electrical conductivity of hydrocarbon films is negligible, which inevitably leads to enormous ohmic components of the measured potential, under external polarization conditions. At the same time, such a picture has never been observed by researchers, when measuring the potentials of structural materials coated with hydrocarbon films in electrolytes aqueous solutions, if partial electrode reactions did not proceed at the limiting current.

A number of phenomena, including the high moisture and oxygen permeability of thin hydrocarbon films (up to $5-6 \cdot 10^{4}$ monolayers), among them, those formed by commercial or used oils or individual hydrocarbons, have been studied quite extensively [5]. 
The purpose of this work was to study the hydrogen cathodic evolution kinetics of iron and copper coated with hydrocarbon films, as well as the anodic dissolution of these metals.

\section{Experimental}

Electrodes, for electrochemical measurements, made of "Armco" iron, with the chemical composition (wt.\%) of C 0.20, Mn 0.51, Si 0.15, P 0.04, S 0.05, Cr 0.32, $\mathrm{Ni} 0.21, \mathrm{Cu} 0.23$, Fe 98.29 and copper M2 with $\mathrm{Al} \leq 0.002, \mathrm{Zn} \leq 0.005, \mathrm{Mn} \leq$ $0.01, \mathrm{Cr} \leq 0.05, \mathrm{Si} \leq 0.01, \mathrm{Zr} \leq 0.05$, and $\mathrm{Cu} 99.7$ were embedded in epoxy resin solidified by polyethylene polyamine, ground with emery paper of varied grades and degreased with acetone or alcohol.

The measurements were carried out at room temperature in aqueous (twice distilled $\mathrm{H}_{2} \mathrm{O}$ ) or methanol solutions with the electrolyte composition of $\mathrm{x} \mathrm{M} \mathrm{HCl}$ + (1 - x) $\mathrm{M} \mathrm{LiCl}(\mathrm{A})$ and $0,1 \mathrm{M} \mathrm{HCl}+\mathrm{x} \mathrm{M} \mathrm{LiCl} \mathrm{+} \mathrm{(3,9} \mathrm{-} \mathrm{x)} \mathrm{M} \mathrm{LiClO}_{4}$ (B), obtained by saturation of the initial solvents with dry hydrogen chloride, up to the specified acidity, and by the addition of lithium chloride dried at $107 \pm 5^{\circ} \mathrm{C}$, and of $\mathrm{LiClO}_{4}$. The salts were of p.a. classification. The solutions with A composition allowed to evaluate the reaction orders for hydrogen ions (dlog $\mathrm{i}_{\mathrm{a}} / \mathrm{d} \log \mathrm{C}_{\mathrm{H}}{ }^{+}$), and with $\mathrm{B}$ compositions, for chloride ions ( $d \log \mathrm{i}_{\mathrm{a}} / \mathrm{d} \log \mathrm{C}_{\mathrm{Cl}}{ }^{-}$), under the assumption that $\mathrm{H}^{+}$and $\mathrm{Cl}^{-}$activity is proportional to their concentration. A part of the studies (the methanol solutions of which dielectric constant value was varied by water addition to alcohol) was carried out on a rotating copper disc electrode, at 1000 rpm. This allowed to completely remove all diffusion limitations which were controlled by the independence of the anodic reaction rate on the disc electrode rotation rate. Potentiostatic measurements, at a potential sweep rate of $0.66 \mathrm{mV} / \mathrm{s}$, were carried out in a three-electrode electrochemical cell made of Pyrex glass working electrode, platinum auxiliary electrode and silver-silver chloride reference electrode, using an IPC-Pro MF potentiostat (produced by the A.N. Frumkin Institute of Physical Chemistry and Electrochemistry of Russian Academy of Sciences). Potentials are given versus the normal hydrogen scale. The investigations on hydrogen evolution reaction (HER) were carried out in a hydrogen atmosphere. Hydrogen overvoltage on the equilibrium hydrogen electrode, in a similar solution, and potentials on the silver-silver chloride reference electrode were measured.

The water contact angle was measured by an «EASY DROP» (KRUSS, Germany) instrument. The hydrocarbon coating thickness $(20 \pm 2 \mu \mathrm{m})$ was gravimetrically estimated, after the film had reached a stationary state. The coatings were formed on the basis of commercial industrial I-20A oil consisting of distillated products of processed low-sulfur petroleum without additives (GOST 20799-88 state standard). Gun grease of the highest quality (GGHQ) (GOST 19537-83) was added to the oil. GGHQ is typically used for metal constructions corrosion protection. 


\section{Results and discussion}

HER kinetics on iron, in hydrocarbon films presence

In acidic aqueous chloride solutions, hydrogen evolution reaction (HER) on iron occurs according to Volmer-Tafel mechanism, with a rate-determining discharge stage [6]:

$$
\begin{array}{ll}
\mathrm{H}_{3} \mathrm{O}^{+}+\mathrm{e} \rightarrow \mathrm{H}_{\mathrm{ads}}+\mathrm{H}_{2} \mathrm{O} & \text { (Volmer reaction) } \\
\mathrm{H}_{\mathrm{ads}}+\mathrm{H}_{\mathrm{ads}} \rightarrow \mathrm{H}_{2} & \text { (Tafel reaction) }
\end{array}
$$

If iron has an oil film coating, the metal hydrogen evolution in an aqueous medium, with a complex chloride composition of electrolyte (A) and $\mathrm{x}=0.05$ 0.99, proceeds in accordance with electrochemical kinetics requirements [7]. However, changes in the rate determining stage nature should not be excluded. Firstly, adsorbed hydrogen removal can proceed, not only, through chemical recombination (2), but also, through the complementary Heyrovsky reaction:

$$
\mathrm{H}_{\text {ads }}+\mathrm{H}_{3} \mathrm{O}^{+}+\mathrm{e} \rightarrow \mathrm{H}_{2}+\mathrm{H}_{2} \mathrm{O}
$$

Secondly, both (2) and (3) stages can be rate-determining.

In order to clarify the rate determining stage nature, HER kinetic parameters, such as $\mathrm{dE} / \mathrm{d} \log \mathrm{i}_{\mathrm{c}}, \mathrm{dE} / \mathrm{d} \log \mathrm{C}_{\mathrm{H}+},\left(\mathrm{d} \log \mathrm{i}_{\mathrm{c}} / \mathrm{d} \log \mathrm{C}_{\mathrm{H}+}\right) \mathrm{E}, \mathrm{d} \eta / \mathrm{d} \log \mathrm{i}_{\mathrm{c}}, \mathrm{d} \eta / \mathrm{d} \log \mathrm{C}_{\mathrm{H}+}$ and $\left(d \log i_{c} / d \log C_{H+}\right)_{\eta}$ (where $i_{c}$ is cathode current density) were evaluated on Armco iron coated with an I-20A oil film containing GGHQ. Their values can be partially calculated on the basis of equations that connect the electrode potential, E, or the hydrogen overvoltage, $\eta$, with the cathode current density, $\mathrm{i}_{\mathrm{c}}$ [7]:

$$
\begin{aligned}
& E=a^{\prime}+\frac{1-\alpha}{\alpha} \Psi_{1}+\frac{R T}{\alpha F} \ln C_{H^{+}}-\frac{R T}{\alpha F} \ln i_{\mathrm{c}} \\
& \eta=a+\frac{1-\alpha}{\alpha} \Psi_{1}-\frac{1-\alpha \frac{R T}{\alpha}}{F} \ln C_{H^{+}}+\frac{R T}{\alpha F} \ln i_{\mathrm{c}^{2}}
\end{aligned}
$$

where a' and a are the constants determined by the electrode material nature, and $\alpha$ is the transfer coefficient, close or equal to 0.5 , for the specific reaction stage. Any different $\alpha$ values are typically associated with barrier-free or non-activation processes. HER kinetic parameters of Armco iron covered with an oil film are summarized in Table 2.

The data in Table 2 show that the I-20A commercial oil film application enforces no changes in HER mechanism. The process was carried on at a rate-determining discharge stage and with a charge transfer coefficient close to 0.5. Diffusion restrictions in the oil film presence were not observed.

$20 \mathrm{wt}$ \% GGHQ addition to the barrier hydrocarbon film fundamentally changed the picture. The process kinetic parameters approach the values corresponding to $\mathrm{HER}$, with the rate-determining stage of chemical recombination. With $\mathrm{C}_{\mathrm{GGHQ}}$ growth in the oil film, or in GGHQ presence, when the kinematic viscosity, $v$, increases by more than 20-30 times, HER kinetics continues to be limited by the reaction (2), without any signs of transport restrictions that could control HER rate. Thus, the hydrated hydrogen $\mathrm{H}_{9} \mathrm{O}_{4}{ }^{+}$ions (taking into account the real hydrate shell), which are rather cumbersome aggregates, pass through a fairly thick hydrocarbon film, without any obstacles. We emphasize here that the oil film is 
formed from a material with a high kinematic viscosity (more than $500 \mathrm{~mm} \cdot \mathrm{s}^{-1}$ ) and a low dielectric constant. Such experimental results make the film continuity doubtful.

Table 2. HER kinetic parameters of iron coated with an I-20A oil film $(20 \pm 2 \mu \mathrm{m}$ thickness) containing GGHQ, in an aqueous solution, with an electrolyte of A composition (room temperature, stationary electrode).

\begin{tabular}{|c|c|c|c|c|c|c|}
\hline 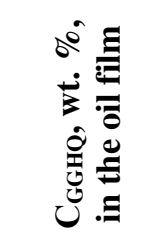 & $-\frac{d E}{d \log i_{\varepsilon}}, V$ & $\frac{d E}{d \log C_{H^{+}}}, V$ & $\left(\frac{d \log i_{\varepsilon}}{d \operatorname{dgg} C_{H^{+}}}\right)_{E}$ & $\frac{d \eta}{d \log i_{c}}, v$ & $-\frac{d \eta}{d \log C_{H^{+}}}, v$ & $\left(\frac{d \log i_{c}}{d \log C_{H^{+}}}\right)$ \\
\hline $\begin{array}{l}\text { without a } \\
\text { film }\end{array}$ & 0.115 & 0.110 & 0.90 & 0.115 & 0.060 & 0.50 \\
\hline 0 & 0.115 & 0.110 & 0.85 & 0.115 & 0.055 & 0.45 \\
\hline 20 & 0.105 & 0.030 & 0.45 & 0.105 & - & 0.20 \\
\hline 40 & 0.105 & 0.040 & 0.35 & 0.105 & 0 & 0 \\
\hline 60 & 0.100 & 0.060 & 0.50 & 0.110 & 0 & 0 \\
\hline 100 & 0.110 & 0.060 & 0.60 & 0.100 & 0 & 0 \\
\hline
\end{tabular}

\section{Copper anodic ionization kinetics in hydrocarbon films presence}

Let us consider the peculiarities of anodic ionization kinetics of metals coated with hydrocarbon films, for instance, copper. The experimental results obtained for this case are especially characteristic.

We first note that copper anodic ionization, in acidic aqueous chloride solutions, has obtained zero ${ }^{\text {th }}$ order for hydrogen ions, and that $\mathrm{n}_{\mathrm{Cl}^{-}}=\mathrm{d} \log \mathrm{i}_{\mathrm{a}} / \mathrm{d} \log \mathrm{C}_{\mathrm{Cl}^{-}}$values are in the range from 2 to 3 [8-10]. When carrying out this experiments, the problem of determining the solvent dielectric constant $(\mathcal{E})$ effect on copper anodic ionization kinetic parameters was first posed. Copper is especially suitable for this process, as an electrode material, since this metal anodic ionization orders, with respect to $\mathrm{H}^{+}$- ions $\left(\mathrm{d} \log \mathrm{i}_{\mathrm{a}} / \mathrm{d} \log \mathrm{C}_{\mathrm{H}}^{+}\right)$, depend on $\varepsilon$. Such data were obtained in the solutions based on the methanol-water solvent. Furthermore, the investigations were carried out by the method of stationary polarization curves in the methanol and water-methanol solutions, which gave rise to $\varepsilon$ variations. The media with the A electrolyte composition (orders on $\mathrm{H}^{+}$to be determined), with an ionic strength of 1, 2.5 and 4, and of B electrolyte (orders on $\mathrm{Cl}^{-}$to be determined) were used in the absence of $\mathrm{ClO}_{4}^{-}$surface activity. To remove the diffusion limitations, studies were carried out on a rotating copper disc electrode (oxygen atmosphere), at 1000 rpm. Methanol dielectric constant is known to be close to 32.6 [11] and, for the methanol solution with $10 \mathrm{wt}$. $\%$ of water, it can be calculated in the first approximation from the following dependence:

$$
\varepsilon_{\text {mixture }}=\varepsilon_{\text {methanol }}+\mathrm{N}_{\text {methanol }}+\varepsilon_{\text {water }}+\mathrm{N}_{\text {water }}
$$


where $\mathrm{N}_{\mathrm{i}}$ is the mole fraction in the true solution, $\varepsilon_{\text {mixture }}$ is approximately equal to 40 and $\varepsilon_{\text {water }}$ is 78 [11]. These solvents $\left(\mathrm{CH}_{3} \mathrm{OH}, \mathrm{H}_{2} \mathrm{O}\right.$ and $\mathrm{CH}_{3} \mathrm{OH}$ with 10 wt. \% $\mathrm{H}_{2} \mathrm{O}$ ) were used to estimate $\varepsilon$ effect on $\operatorname{dlog} i_{a} / \mathrm{d} \log C_{\mathrm{Cl}^{-}}$value, taking into account a total $\mathrm{HCl}$ electrolytic dissociation and $\mathrm{dlog} \mathrm{i}_{\mathrm{a}} / \mathrm{d} \log \mathrm{C}_{\mathrm{H}}{ }^{+}$value equal to zero, in the aqueous medium. As it was shown by independent experiments, the effective charge of copper cations passing into the solution, under the studied conditions, is 1.

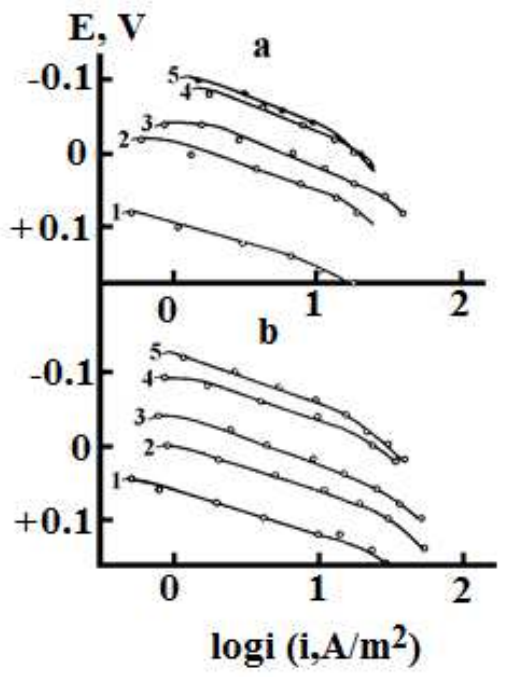

Figure 1. Copper stationary anodic polarization curves in anhydrous (a) methanol solutions and methanol solutions with $10 \% \mathrm{H}_{2} \mathrm{O}$, and (b) with an electrolyte composition of $0.1 \mathrm{M} \mathrm{HCl}+\mathrm{y} \mathrm{M} \mathrm{LiCl}+(2.9-\mathrm{y}) \mathrm{M} \mathrm{LiClO} 4 \cdot \mathrm{y}, \mathrm{mol} / \mathrm{L}: 1-0,1 ; 2-0.5,3-1.0 ; 4-$ $2.7 ; 5-3.89$.

The character of the anodic polarization curves obtained for copper in the methanol solutions is shown in Fig. 1.

Copper anodic dissolution kinetic parameters, obtained under these conditions, are given in Table 3.

Table 3. Copper ionization anodic reaction order, with respect to dlog $\mathrm{i}_{\mathrm{a}} / \mathrm{d} \log \mathrm{CH}_{\mathrm{H}}{ }^{+}\left(\mathrm{n}_{\mathrm{H}}{ }^{+}\right)$ hydrogen ions and dlog $i_{a} / d \log \mathrm{C}_{\mathrm{Cl}^{-}}\left(\mathrm{n}_{\mathrm{Cl}^{-}}\right)$chlorine ions and $\mathrm{dE} / \mathrm{d} \log \mathrm{i}_{\mathrm{a}}\left(\mathrm{b}_{\mathrm{a}}\right)$ of anode curves Tafel sections, in methanol and water-methanol solutions.

\begin{tabular}{|c|c|c|c|c|c|c|c|c|}
\hline \multirow{3}{*}{$\begin{array}{l}\text { Solution } \\
\text { ionic } \\
\text { strength }\end{array}$} & \multicolumn{4}{|c|}{$N_{\text {methanol }} \sim 1,0(\varepsilon=32,6)$} & \multicolumn{4}{|c|}{$N_{\text {methanol }} \sim 0,835(\mathcal{E} \approx 40)$} \\
\hline & \multicolumn{2}{|c|}{$\begin{array}{l}\text { A chloride } \\
\text { electrolyte }\end{array}$} & \multicolumn{2}{|c|}{$\begin{array}{l}\text { B chloride- } \\
\text { perchlorate electrolyte }\end{array}$} & \multicolumn{2}{|c|}{$\begin{array}{l}\text { A chloride } \\
\text { electrolyte }\end{array}$} & \multicolumn{2}{|c|}{$\begin{array}{l}\text { B chloride- } \\
\text { perchlorate } \\
\text { electrolyte }\end{array}$} \\
\hline & $\eta_{\mathbf{H}^{+}}$ & $\begin{array}{l}b_{\mathrm{a}} \\
\mathbf{V} \\
\end{array}$ & $\eta_{\mathrm{c} l-}$ & $\begin{array}{l}b_{\mathrm{a}} \\
\mathbf{V}\end{array}$ & $\eta_{\mathbf{H}^{+}}$ & $\begin{array}{l}b_{\mathbf{a}} \\
\mathbf{V} \\
\end{array}$ & $\eta_{\mathrm{cl}-}$ & $\begin{array}{l}\boldsymbol{b}_{\mathrm{a}} \\
\mathbf{V} \\
\end{array}$ \\
\hline 1.0 & 2.2 & 0.060 & - & - & 0.4 & 0.065 & - & - \\
\hline 2.5 & 1.9 & 0.060 & - & - & - & - & - & - \\
\hline 4.0 & 2.3 & 0.060 & 2.7 & 0.060 & 1.9 & 0.065 & 2.7 & 0.060 \\
\hline
\end{tabular}


As it follows from Table 3 , in the anhydrous methanol solutions, $\mathrm{n}_{\mathrm{H}}{ }^{+}$is greater than zero, decreasing with water addition. In the aqueous media, as noted above, $\mathrm{n}_{\mathrm{H}}^{+}=0$. Since $\mathrm{H}^{+}$ions are superficially inactive, their participation in the electrode process can be explained by the existence of ionic twins and tees formed at a $\varepsilon$ low value, especially in a double electric layer.

The obtained kinetic parameters are satisfactorily interpreted by the following copper ionization mechanism. The first stage has the following forms:

$$
\begin{aligned}
& \mathrm{Cu}+\mathrm{Cl}^{-} \leftrightarrow(\mathrm{CuCl})_{\mathrm{ads}}+\mathrm{e} \\
& \mathrm{Cu}+\mathrm{H}^{+} \mathrm{Cl}^{-} \leftrightarrow(\mathrm{CuHCl})^{+}{ }_{\mathrm{adc}}+\mathrm{e} \\
& \mathrm{Cu}+\mathrm{H}^{+} \mathrm{Cl}^{-} \mathrm{H}^{+} \leftrightarrow\left(\mathrm{CuH}{ }_{2} \mathrm{Cl}\right)^{2+}{ }_{\mathrm{adc}}+\mathrm{e},
\end{aligned}
$$

or generalized

$$
\mathrm{Cu}+\left(\mathrm{H}_{n} \mathrm{Cl}_{l}\right)^{\mathrm{z} \leftrightarrow}\left(\mathrm{CuH}_{n} \mathrm{Cl}_{l}\right)_{\mathrm{adc}}{ }^{(\mathrm{z}+1)}+\mathrm{e}
$$

where $z$ and $z+1$ are charges of ion formation, and the intermediate adsorption complex, respectively, and $\mathrm{n}$ varies from 0 to 2 . Then, the rate determining chemical stage is the following:

$$
\left(\mathrm{CuH}_{n} \mathrm{Cl}_{l}\right)_{\mathrm{ads}}^{z+1}+p \mathrm{Cl}^{-} \leftrightarrow\left(\mathrm{CuCl}_{1+p}\right)^{-(1+p-1)}+n \mathrm{H}^{+}
$$

For the rate determining stage we write the kinetic equation:

$$
i_{7}=k_{7} C_{\left(\mathrm{CuH}_{n} \mathrm{Cl}_{2}\right)^{z+1}} \mathrm{C}_{\mathrm{Cl}^{-}}^{p}
$$

It is not difficult to show that the kinetic equation of copper anodic ionization process takes the following form:

$$
i_{\mathrm{a}}=k C_{\mathrm{H}^{+}}^{n} C_{\mathrm{Cl}^{-}}^{p+1} \exp (E F / R T)
$$

At low $\mathrm{HCl}$ concentrations, the ion twins and tees do not play any important role $\left(\mathrm{n}_{\mathrm{H}}{ }^{+}=0\right) . \mathrm{n}_{\mathrm{H}}^{+}=2$ value indicates that the process (6) dominates. Summarizing the above said, one can write down

$$
i_{\mathrm{a}}=\left(k_{4}+k_{5} C_{\mathrm{H}^{+}}+k_{6} C_{\mathrm{H}^{+}}^{2}\right) C_{\mathrm{cl}^{-}}^{p+1} \exp (E F / R T)
$$

Based on $\mathrm{n}_{\mathrm{Cl}}$ value, $\mathrm{p}$ is equal to 1 or 2 .

Water addition helps to destroy the ionic twins and tees, especially in the solutions with low ionic strength [12]. Therefore, in the presence of a mole fraction of $\mathrm{H}_{2} \mathrm{O}$ equal to $0.165,0<\mathrm{n}_{\mathrm{H}}{ }^{+}<1$ inequality is observed.

When $\mathrm{k}_{4} \gg\left(\mathrm{K}_{5} \mathrm{C}_{\mathrm{H}^{+}} \mathrm{k}_{5}+\mathrm{k}_{6} \mathrm{C}_{\mathrm{H}^{+}}{ }^{+}\right)$the zero ${ }^{\text {th }}$ order on hydrogen ions is expected; when $\mathrm{k}_{4} \approx \mathrm{k}_{5} \mathrm{C}_{\mathrm{H}^{+}}$and $\mathrm{k}_{4}>>\mathrm{k}_{6} \mathrm{C}_{\mathrm{H}^{+}}^{2}$, one obtains $0<\mathrm{n}_{\mathrm{H}}{ }^{+}<1$. As it follows from these results, singly-charged copper ions exist in chloride methanol solutions, as $\mathrm{CuCl}_{2}{ }^{-}$ and $\mathrm{CuCl}_{3}{ }^{2-}$ complex ions, which are known to take place in water too.

In the course of further research, it was important to find out whether copper anodic ionization mechanism, in the active state, would change if protective oil films were applied. To this end, an investigation on copper kinetics and dissolution mechanism, under similar conditions, was undertaken. Carrying out studies on a rotating disc electrode covered with a hydrocarbon film is impossible, because the barrier oil layer slides off the metal surface, due to rotation. The following process parameters were evaluated: $\mathrm{dE} / \mathrm{d} \log \mathrm{i}$ (Tafel slope coefficient), reaction orders for hydrogen cations $\left(d \log \mathrm{i}_{\mathrm{a}} / \mathrm{d} \log \mathrm{C}_{\mathrm{H}}^{+}\right)$and chlorine ions $\left(\mathrm{d} \log \mathrm{i}_{\mathrm{a}} / \mathrm{d} \log \mathrm{C}_{\mathrm{Cl}}^{-}\right.$), where $\mathrm{i}_{\mathrm{a}}$ is the anode current density. To this end, stationary potentiostatic anodic polarization 
curves were measured in the solutions with a complex electrolyte composition, similar to that of methanol media. However, a stationary electrode covered with the hydrocarbon film of the specified composition was used, because of the stated above reasons.

The character of the anodic polarization curves, obtained on copper coated with an I-20A oil film with 40 and 60 wt.\% GGHQ, is shown in Fig. 2.
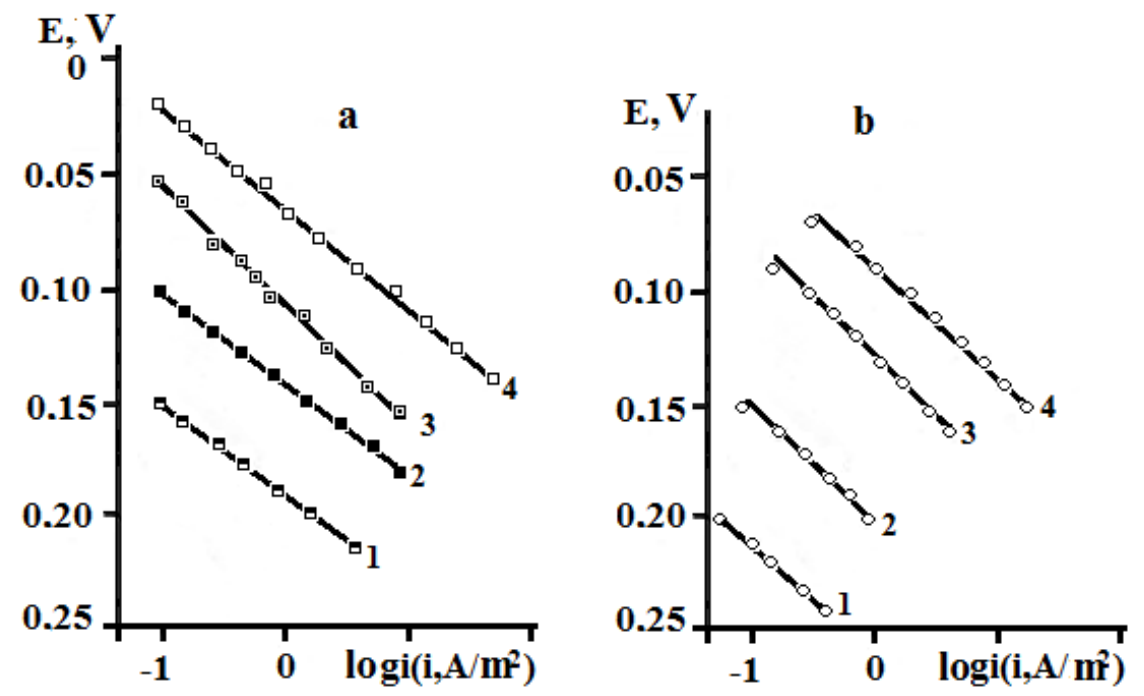

Figure 2. Stationary anodic polarization curves of copper coated with an oil film containing GGHQ, in aqueous solutions with an electrolyte composition of: (a) $0.1 \mathrm{M}$ $\mathrm{HCl}+\mathrm{y} \mathrm{M} \mathrm{LiCl}+(2.9-\mathrm{y}) \mathrm{M} \mathrm{LiClO} 4 \cdot \mathrm{y}, \mathrm{mol} / \mathrm{L}: 1-0,1 ; 2-0.5,3-1.5 ; 4-2.5$; CGGHQ, wt.\%: 40; and of (b) 60.

As it follows from Fig. 2, all the anodic polarization curves have sufficient lengths of the segments, with a Tafel slope coefficient of $\mathrm{dE} / \mathrm{d} \log \mathrm{i}_{\mathrm{a}}$ equal to $0.060 \mathrm{~V}$. This reveals copper ionization in the form of singly charged ions.

Copper anodic dissolution kinetic parameters are given in Table 4.

Table 4. Anodic dissolution kinetic parameters of copper coated with a hydrocarbon film based on I-20A oil, with 40 and 60 wt. \% of GGHQ.

\begin{tabular}{|c|c|c|c|}
\hline \multirow{2}{*}{ Parameter } & \multicolumn{3}{|c|}{$C_{\mathrm{GGHQ}}$ in the coating } \\
& wt. \% & 60 \\
\cline { 2 - 4 } & Coating absence & 40 & 0 \\
\hline$d \log i_{d} / d \log C_{H}{ }^{*}$ & 0 & 0 & 2.2 \\
$d \log i_{a} / d \log C_{C l}^{-}$ & 3.0 & 2.2 & \\
\hline
\end{tabular}

It is of fundamental importance that $d \log \mathrm{i}_{\mathrm{a}} / \mathrm{d} \log \mathrm{C}_{\mathrm{H}^{+}}$quantity, under the studied conditions, is equal to zero, similar to that of an aqueous medium without an oil coating. This means that copper ionization takes place at the interface between copper/liquid phases, with a high $\varepsilon$ value. Thus, it should be assumed that the anodic process is taking place in an aqueous medium or in a system rich in water. 
Anyway, its $\varepsilon$ value is appreciably greater than 40 , which reveals the presence of an aqueous medium, at the boundary with the copper surface coated with a hydrocarbon film.

\section{Additional evidence of hydrocarbon films porosity}

There are other experimental results that reveal hydrocarbon films complex structure. Thus, in addition to water molecules [3] and $\mathrm{SO}_{2}$ [4], large organic molecules, in particular, oxyethylated amines, with the conditional molecular formula given below, pass almost unimpeded through hydrocarbon films:

$$
\mathrm{R}-\mathrm{N}-\left(\mathrm{CH}_{2} \mathrm{CH}_{2} \mathrm{O}\right)_{x} \mathrm{H}
$$

In this formula, $x+y$ is equal to 2,5 or 14 and $\mathrm{R}$ is $\mathrm{C}_{17} \ldots \mathrm{C}_{20}$.

To establish a similar fact, these compounds were introduced into corrosion protective hydrocarbon films, in some cases, for iron or copper, and in the others, in a comparable concentration, directly into the corrosive medium (solution). In the latter cases, the original oil films did not contain specially introduced inhibitors or these were initially absent at the corroding metal surface. In all cases, potentiostatic polarization curves were measured. The corresponding curves are shown in Fig. 3, and the values of the protective effect are shown in Table 5.

Regardless of $x+y$ value, oxyethylated amine (OEA) presence, in a film or corrosive medium, sharply inhibits the anodic reaction on iron, in a $1 \mathrm{M} \mathrm{HCl}$ solution. Moreover, the corrosion protective effect of the inhibitor introduced into the acidic solution was observed immediately after the electrode immersion in the solution, when the inhibitor molecules supply to the corroding surface, through the oil film, has been carried out at a high rate, which the mass transfer process is unlikely to cause. It should be assumed that the dissolved inhibitor approaches the metal surface as a part of the solution. In this case, the uninhibited oil film did not practically change the electrode reactions kinetics, in OEA absence, in the liquid phase volume (Fig. 3, curves 1 and 2), and, apparently, did not practically change its mechanism. In the inhibited solution (Fig. 3, curves 3-8), a similar pattern has been observed, so that the existence of the oil film negligibly affects the electrode reactions kinetics. At the same time, a relative disposition thereof proves the existence of an anodic process inhibition, compared to that of the non-inhibited solution. Hence, the inhibitor in situ inhibits the anodic process at the electrode surface, both with and without an oil film; that is, the inhibitor freely penetrates through the oil film towards the metal surface. 


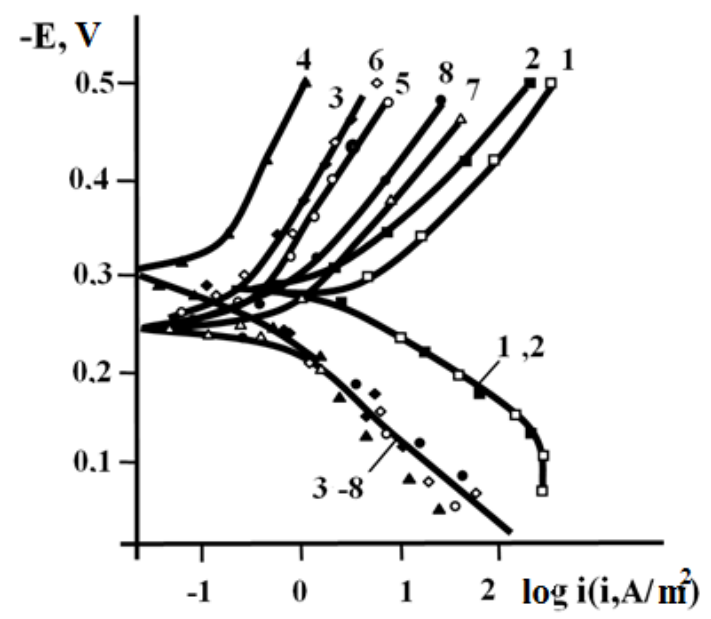

Figure 3. Potentiostatic polarization curves of iron in uninhibited $(1,2)$ and inhibited $(1$ $\mathrm{g} / \mathrm{L}$ oxyethylated amines) $1 \mathrm{M} \mathrm{HCl}$ solutions $(3-8) .1,3,5,7$ - no protective film; 2, 4, 6,8 - the electrode is coated with an I-20A oil film of $20 \mu \mathrm{m}$ in thickness. Number of oxyethyl groups in the inhibitor molecule:3, 4-2; 5, 6-5; and 7, 8-14.

The inhibitor effect on the cathodic reaction kinetics is more selective compared to that of iron anodic ionization, but the observed tendency is completely preserved. The nature of OEA effect on partial electrode reactions kinetics, at various inhibitor input routes, correlates satisfactorily with the nature of the additive effect on M2 copper corrosion rate, in various media (Table 5).

Table 5. Dependence of copper electrochemical corrosion rate on OEA $(x+y=14)$, and its protective effect $(\mathrm{Z}, \%)$, at a concentration of $0.1 \mathrm{wt} . \%$, in the solution or surface oil coating (coating thickness is $10 \mu \mathrm{m}$, at air).

\begin{tabular}{|c|c|c|c|c|}
\hline $\begin{array}{c}\text { Electrolyte solution } \\
\text { composition }\end{array}$ & $\begin{array}{c}\text { Phase where the } \\
\text { inhibitor is present }\end{array}$ & $\begin{array}{c}\text { Protective coating } \\
\text { composition }\end{array}$ & $\boldsymbol{i}_{\text {cor }} \cdot \mathbf{1 0}^{\mathbf{5}} \mathbf{A} / \mathbf{c m}^{\mathbf{2}}$ & $Z, \%$ \\
\hline \multirow{4}{*}{$0.5 \mathrm{M} \mathrm{NaCl}$} & solution & - & 14.1 & - \\
\cline { 2 - 5 } & solution & I-20A & 1.0 & 93 \\
\cline { 2 - 5 } & coating & I-20A+OEA & 1.5 & 89 \\
\cline { 2 - 5 } & solution & OEA & 1.1 & 92 \\
\hline \multirow{3}{*}{$0.5 \mathrm{M} \mathrm{NaCl}+0.1 \mathrm{M} \mathrm{HCl}$} & solution & I-20A & 0.4 & - \\
\cline { 2 - 5 } & solution & I-20A+OEA & 0.8 & 85 \\
\cline { 2 - 5 } & coating & OEA & 2.2 & 58 \\
\cline { 2 - 5 } & solution & & & \\
\end{tabular}

The results show that oil compositions are better wetting agents for iron and copper surfaces than water and aqueous solutions (Table 1). Because of this, as noted earlier, such compositions must efficiently displace surface phase aqueous layers of which existence at the hydrocarbon film/metal interface becomes highly questionable. On the other hand, without $\mathrm{H}_{2} \mathrm{O}$ molecules presence and participation, metal corrosion cathodic and anodic reactions, with protective oil films, should be substantially suppressed, and the process rate, as a whole, is 
expected to tend to zero, which does not seem to have been experimentally confirmed.

Also, the dielectric constant of the liquid phase (LP), close to 2, is not at the LP /metal surface, since a sufficiently high LP electrical conductivity is observed. At the solid/liquid interface, there is a double electric layer, without which both metals electrochemical corrosion and partial electrode reactions are impossible.

These experimental data can be consistently explained if one accepts discontinuities (capillaries) presence in hydrocarbon films. A schematic view of such capillaries (pores) is shown in Figure 4.

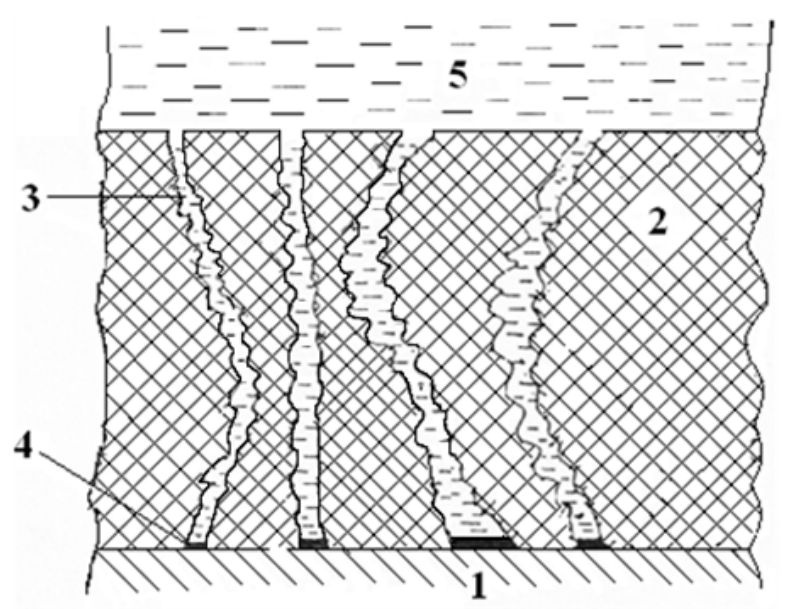

Figure 4. Schematic representation of the oil film/metal interface: 1 - metal surface; 2 - oil film; 3 - channel (discontinuity); 4 - double electrical layer; and 5 aqueous solution.

The total cross section of such discontinuities depends on the nature and, especially, on the surfactant concentration, decreasing with its increase. Probably, the actual cross section of individual discontinuities is similar, as noted earlier, on a first approximation, to channels (pores) that penetrate the film (Fig. 4), normally, tangentially, or at any arbitrary angle relative to the metal surface, and it can be represented in this way. Most likely, these channels are complex, as their existence and their configurations are not constant in time. If the channels are not filled with the solution, a continuous stochastic sticking of their walls is possible, with the discontinuities destruction and formation of new ones. However, the discontinuities total cross section - with a constant film composition - and the conditions of its existence remain practically unchanged in time, resulting in $\mathrm{dm}_{\mathrm{H}_{2} \mathrm{O}} / \mathrm{d} \tau=$ const. The water can be supplied through these channels, in the form of vapor, but the film discontinuities are more likely to be filled with an aqueous corrosive-aggressive medium, in the form of an independent phase. Therefore, the question on the water supplying mechanism is no longer relevant. Another indirect proof of the pores presence in oil films is a sufficiently high rate of water supply to the corroding surface. This cannot be attributed to the true water solubility in the oil film, since it is very small. Another hypothesis of water supply to the corroding 
surface would be the mass transfer of liquid droplets, in the case of water-in-oil type micellar formations. The movement of such relatively large formations is quite possible. However, if their size would reach hundreds of nanometers, their ability to pass through discontinuities in oils, among them, those thickened with additives, would require additional assumptions. It is difficult to imagine formations of such a size, although, in the case of oil films, specific secondary effects remain possible.

\section{Conclusion}

Hydrocarbon films deposited on iron and copper do not change the hydrogen evolution reaction mechanism (on $\mathrm{Fe}$ ) and metals anodic ionization $(\mathrm{Cu}, \mathrm{Fe})$, in acidic chloride solutions. On Armco iron, in the presence of a hydrocarbon film, HER proceeded through successive stages of discharge (Volmer reaction) and chemical recombination (Tafel reaction). There is only a transition from the delayed first stage (in the absence of a coating) to the second one.

Copper anodic ionization kinetics, when hydrocarbon films are applied to its surface, remains unchanged, which reveals the presence of an aqueous phase, under the oil coating surface. This indicates discontinuities, in the form of channels (capillaries) containing an aqueous solution, which penetrate the coating. Hydrocarbon films are highly permeable to substances characterized by large molecules, in particular, oxyethylated amines with up to 14 oxyethyl groups that are dissolved in the acidic aqueous chloride solutions. Moreover, OEA molecules reach the metal surface (iron) and change the partial electrode reactions kinetics, immediately after the electrodes immersion in the solution. No continuity of the protective oil films is assumed, and the total cross-section of the penetrating capillaries depends on the films components nature and concentration.

\section{Acknowledgments}

The study was supported by the Russian Science Foundation, Project No. 18-16-00006.

\section{References}

1. Shekhter $\mathrm{Yu}$ N. Metal protection against corrosion (inhibitors, oils, lubricants). Moscow:Khimiya; 1964.

2. Groysman A. Corrosion in Systems for Storage and Transportation of Petroleum Products and Biofuels (Identification, Monitoring and Solutions). Heidelberg: Springer; 2014.

3. Vigdorovich VI, Tsygankova LE, Shel NV, et al. Protection of metals against atmospheric corrosion by oil coatings (theory, practice, ecological aspects). CARTEC. Moscow. 2014.

4. Tsygankova LE, Vigdorovich VI, Shel NV, et al. Inhibited oil coatings for anticorrosion protection of carbon steel in atmosphere containing $\mathrm{SO}_{2}$. Farby $\mathrm{i}$ Lakiery (Paints and Varnishes). 2018;26:35-41. 
5. Vigdorovich VI, Tsygankova LE, Tanygina ED, et al. Anticorrosion thin-film materials on base of individual paraffinic hydrocarbons. Pershin Tambov. 2013.

6. Vigdorovich VI, Tsygankova LE, Balybin DV, et al. Influence of ofluorpbenylbiguanidin on the kinetics of hydrogen evolution reaction on iron, the nature of rate-determining step and hydrogen diffusion through a steel membrane. J Electroanal Chem. 2013;689:117-23. http://dx.doi.org/10/1016/j/jelechem.2012.10.021

7. Frumkin AN. Selected Works: Electrode Processes. Moscow: Nauka; 1987.

8. Lee HP, Nobe $\mathrm{K}$. Kinetics and mechanisms of $\mathrm{Cu}$ electrodissolution in chloride media. J Electrochem Soc. 1986;133:2035-2043. https://doi.org/10.1149/1.2108335

9. Meng Y, Bard AJ. Measurement of temperature-dependent stability constants of $\mathrm{Cu}(\mathrm{I})$ and $\mathrm{Cu}(\mathrm{II})$ chloride complexes by voltammetry at a $\mathrm{Pt}$ ultramicroelectrode. Anal Chem. 2015;87:3498-3504. https://doi.org/10.1021/acs.analchem.5b00052

10. Li S, Teague MT, Doll GL, et al. Interfacial Corrosion of Copper in Concentrated Chloride Solution and the Formation of Copper Hydroxychloride. Corros Sci. 2018;141:243-254. https://doi.org/10.1016/j.corsci.2018.06.037.

11. Kiper RA. Physical properties of substances. Handbook of Chemistry. Khabarovsk; 2013.

12. Bacarella AL, Griss JC. The Anodic Dissolution of Copper in Flowing Sodium Chloride Solutions Between $25^{\circ}$ and $175^{\circ} \mathrm{C}$. J Electrochem Soc. 1973;120:459-465. https://doi.org/10.1149/1.2403477 